\title{
Multileveled tunable silicon grating array bonded on large scale integration substrate by polymers
}

\author{
Tomohiro Suzuki, ${ }^{a}$ Chernroj Sawasdivorn, ${ }^{a}$ Takashi Sasaki, ${ }^{a}$ \\ Hiroshi Matsuura, ${ }^{b}$ and Kazuhiro Hane ${ }^{a}, *$ \\ ${ }^{a}$ Tohoku University, Department of Fine Mechanics, Sendai, Japan \\ ${ }^{\mathrm{b}}$ Tohoku Gakuin University, Department of Mechanical Engineering, Tagajo, Japan
}

\begin{abstract}
A multileveled tunable silicon grating array is designed and fabricated on a largescale-integration (LSI) substrate. The grating consists of 250 single crystalline silicon ribbons of $260 \mathrm{~nm}$ in thickness, $400 \mu \mathrm{m}$ in length, and $10 \mu \mathrm{m}$ in period. The LSI substrate generates voltages to vary the heights of respective grating ribbons addressed by digital signal input. Each grating ribbon is attracted by an electrostatic force generated by 6-bit applied voltage in the range from 0 to $5 \mathrm{~V}$. In the fabrication, the LSI substrate and a silicon-on-insulator wafer are bonded by the two kinds of polymers. A photosensitive polyimide polymer is used for patterning the bonding pads and also for bonding the wafers at the pressure of $0.25 \mathrm{MPa}$ and the temperature of $350^{\circ} \mathrm{C}$. Another epoxy polymer fills the space underneath the grating ribbons for subsequent process, which is finally removed by sacrificial etching to make the grating ribbons freestanding. The tunability of the grating is examined experimentally under the basic operation conditions. (C) The Authors. Published by SPIE under a Creative Commons Attribution 4.0 Unported License. Distribution or reproduction of this work in whole or in part requires full attribution of the original publication, including its DOI. [DOI: 10.1117/1.JOM.1.1.013501]
\end{abstract}

Keywords: micro-optics; optical MEMS; grating; polymer bonding; silicon on insulator.

Paper 20008 received Sep. 2, 2020; accepted for publication Oct. 29, 2020; published online Jan. 26, 2021.

\section{Introduction}

Diffraction grating is an important optical component in spectroscopic optical systems. In particular, blazed grating is efficient because a strong intensity is obtained only in the first-order diffraction. The cross-sectional profile of the blazed grating usually has a saw-toothed shape. Grating profile can be tuned using microelectromechanical systems (MEMS) technology. Several tunable gratings, i.e., the pitch-variable gratings ${ }^{1-4}$ and diffraction-efficiency-tunable gratings, ${ }^{5-7}$ were reported. In the intelligent tunable grating systems, each reflection element of grating, such as a narrow ribbon and a small square mirror, is independently controlled by each actuation force, which produces a high degree of freedom in grating profile as a function of time. ${ }^{8-11}$ The grating ribbons in air go down alternately to generate the switchable diffraction beam. ${ }^{8}$ The switchable diffraction is successfully utilized for image projection (known as grating light valves). ${ }^{8,9}$ In addition, the grating ribbons are controlled continuously in their heights for correlation spectroscopy as polychromator ${ }^{11}$ and for wavelength management (Polychromix, Inc.). More recently, the high-speed switching of an optical phased array has been demonstrated using the addressable subwavelength high-contrast grating mirrors. ${ }^{10}$

One of the promising applications of switchable diffraction is the wavelength selective switch (WSS) for fiber-optic telecommunication because of the continuous increase of routing data. ${ }^{12}$ Liquid crystal on silicon was proposed to generate a diffraction beam for switching. ${ }^{13,14}$ Instead of the refractive index change, the periodic height distribution of reflection elements generates a controllable diffraction beam using MEMS technology. The periodic height distribution of micromirrors can be formed by the piston-motions of micromirror. ${ }^{10,11,15}$

The integration of large-scale-integration (LSI) circuit with micromirrors is useful for a large scale addressable micromirror array to generate a phase-distribution of reflection grating. In

*Address all correspondence to Kazuhiro Hane, hane2@hane.mech.tohoku.ac.jp 
general, the integration of LSI circuits with MEMS is promising for advanced microsystems. MEMS made of materials different from silicon can be combined with LSI by stacking their wafers for heterogeneously integrated devices such as intelligent and autonomy sensors. On the other hand, the integration of LSI circuit with MEMS is often restricted by fabrication conditions such as material and temperature. ${ }^{16,17}$ Several silicon membrane transfer bonding technologies for LSI-MEMS integration have been reported. ${ }^{16}$ Among them, polymer-based bonding process is preferred for transferring the single crystal silicon membrane on LSI circuit at low temperature. ${ }^{18}$ The torsional micromirror array was bonded on the driving LSI circuit for light modulator using a thermosetting polymer $(\mathrm{BCB}) .{ }^{19}$ The polyimide-based polymer membrane transfer bonding was also used for the fabrications of a radiofrequency filter ${ }^{20}$ and a grating array on glass substrate. ${ }^{21}$ However, there are very few reports on the integration of MEMS grating with LSI substrate.

In this paper, a multileveled tunable silicon grating array is designed and fabricated on an LSI substrate using two-step polymer bonding method. The LSI substrate and a silicon-on-insulator (SOI) wafer are bonded by the two kinds of polymers at the temperatures lower than $350^{\circ} \mathrm{C}$ and the pressure of $0.25 \mathrm{MPa}$. The LSI substrate generates the voltages to vary the heights of the respective grating ribbons addressed by digital signal input. The tunability of the grating is tested experimentally under the basic operation conditions.

\section{Principle and Design}

Figure 1(a) shows a cross-sectional schematic diagram of the proposed multileveled tunable grating. The tunable grating consists of a number of ribbons in air fixed at their both-ends. The heights of the ribbons are changed by the respective electrostatic forces generated from the voltage applied to the electrodes of LSI circuits. The height profile can be adjusted to be a saw-toothed shape for obtaining a blazed reflection grating when the applied voltages to the electrodes are arranged to decrease gradually and periodically.

Here, we assume that the grating position is linearly decreased as shown in Fig. 1(a) in a period of $P$. When the number of ribbons (the number of levels in height) in a period is denoted by $m$, the first-order diffraction intensity becomes the highest under the condition that the ribbons are placed at the respective positions so that the one cycle phase $2 \pi$ is divided into $m$ equally spaced phases given by a function $f(x)$ with $x$ denoting the position vertical to grating ribbons. The diffraction efficiency in the respective orders are calculated using the complex Fourier series expansion. ${ }^{22}$ The first-order diffraction coefficient $C_{1}$ is expressed as

(a)

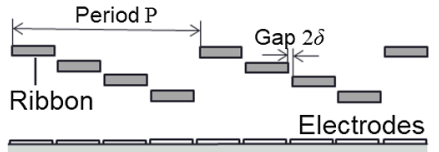

(c)

Tunáble grating

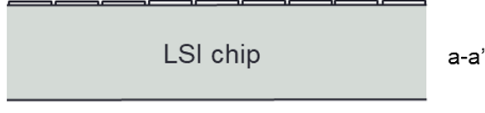

(b)

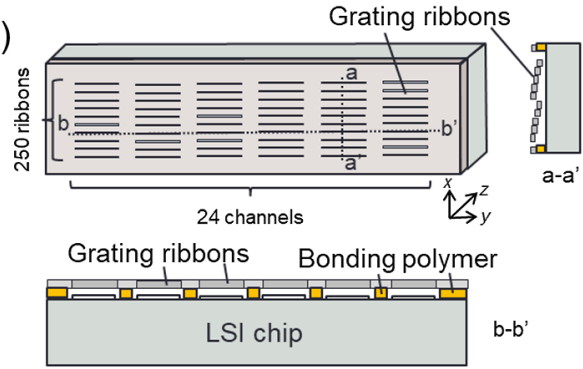

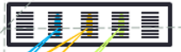

Fig. 1 (a) Cross-sectional schematic of multileveled tunable grating, (b) arrangement of grating array, and (c) optical system for the application to WSS. 


$$
C_{1}=\frac{1}{P} \int_{0}^{P} f(x) e^{-\frac{i 2 \pi x}{P}} \mathrm{~d} x
$$

The function $f(x)$ expresses the reflection phases form $m$-leveled grating ribbons as

$$
f(x)=\alpha \exp \left(\frac{i 2 \pi k}{m}\right), \quad\left(P \frac{k}{m}+\delta \leq x \leq P \frac{k+1}{m}-\delta, k=0,1, \ldots m-1\right) .
$$

Here, $2 \delta$ is the gap between grating ribbons and $\alpha$ is the amplitude reflectance of grating ribbons. Then,

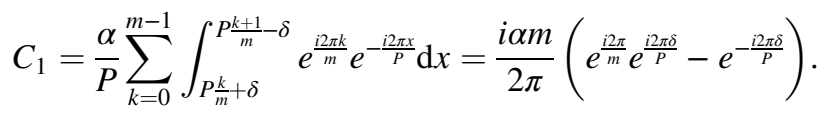

For the $m$-leveled grating with a period of $P$, the intensity normalized by the incident light intensity is expressed by the square of the absolute value of $C_{1}$

$$
\left|C_{1}\right|^{2}=\frac{\alpha^{2} m^{2}}{2 \pi^{2}}\left[1-\cos 2 \pi\left(\frac{1}{m}+\frac{2 \delta}{P}\right)\right] .
$$

The first-order diffraction is obtained at the diffraction angle $\theta$ of $\sin \theta=\frac{\lambda}{P}$ for the vertically incident beam at a wavelength $\lambda$. The diffraction angle corresponding to the steering angle can be changed by varying the grating period $P$.

Although the purpose of this paper does not complete a WSS, the basic design of the WSS is briefly described below. In order to design the WSS using the multileveled tunable gratings, we consider a tunable grating array operated by LSI circuits as shown in Fig. 1(b). The basic optical configuration of the WSS is also shown in Fig. 1(c). If we consider manipulation of 24 wavelength division multiplexed signals, 24 gratings are needed to switch the wavelength divided signals into 24 output fibers. Generating $m$-leveled grating ribbons in a period $P[=m(w+2 \delta)]$, the deflection angle $\theta$ of the first-order diffraction beam is given by the equation $\sin \theta=\lambda /[m(w+2 \delta)]$, where $w$ is the width of the grating ribbon. Using the transforming lens with a focal length of $f$, the vertical position $x_{f}$ on the output fiber plane is approximately $x_{f} \cong f \lambda /[m(w+2 \delta)]$. Selecting $f$ to be around $200 \mathrm{~mm}, 12$ output fibers are aligned at the upper side with a space larger than the fiber diameter $(125 \mu \mathrm{m})$ using $m$-leveled blazes grating from $m=3$ to $m=15$. The blaze direction is switched from the upper side to the lower side on the output plane. Under the condition of $m=15$, the number of periods is 16.7 in the grating consisting of 250 grating ribbons. The details of the design of the WSS may be described elsewhere.

Figure 2 shows the schematic diagram of a custom-made LSI for controlling the multileveled gratings. The maximum voltage generated at the electrodes of grating is $5 \mathrm{~V}$. The voltage division is 6 bits corresponding to the minimum voltage step of $78 \mathrm{mV}$. The LSI consists of the serial/ parallel converters with five input electrodes, memories, DA converters, etc. as shown in Fig. 2. In the case of a clock frequency of $4 \mathrm{MHz}$, the necessary time to set up a voltage of an electrode for electrostatic actuation is $\sim 10 \mu$ s using the SPI communication. In order to update the voltages of 250 grating ribbons, it takes $2.5 \mathrm{~ms}$. And thus, the total time for setting the grating ribbons of 6000 in 24 channels is about $60 \mathrm{~ms}$. Therefore, the update time of grating is dependent on the number of the grating ribbons to be set up. If the clock time is increased to $25 \mathrm{MHz}$, the total time for setting the grating array is decreased to be $10 \mathrm{~ms}$.

Figure 3(a) shows the designed structure of the multileveled tunable silicon grating, which is decomposed to show the layered structures. The top grating layer is made of the top silicon layer of a SOI wafer with $1-\mu \mathrm{m}$ thick buried oxide layer. The thickness of the top silicon layer is $260 \mathrm{~nm}$. The top silicon layer with gratings is located above the electrodes of LSI substrate after bonding with the first polymer (photosensitive polyimide polymer). The air gap between the grating ribbon and the electrode is designed to be $2.6 \mu \mathrm{m}$. The regions between the gratings are bonded by the polymer, i.e., grating support shown in Fig. 3(b). Moreover, the top silicon layer is bonded at the both ends by the bonding pads formed by the polymer as shown in 


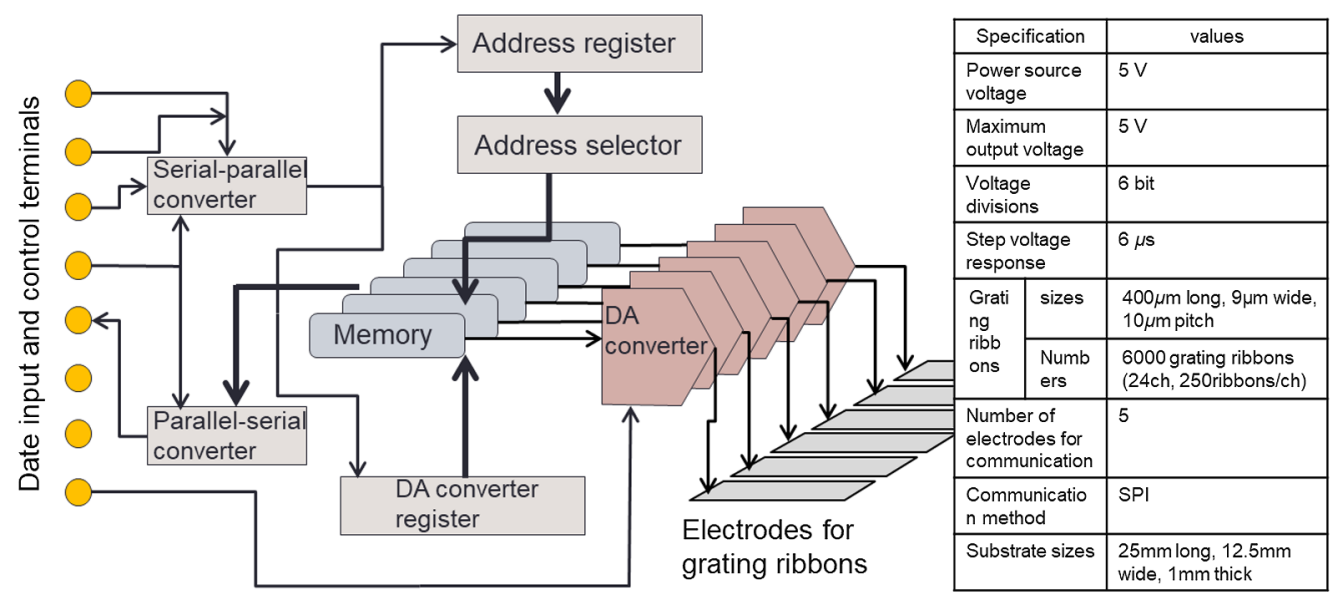

Fig. 2 Schematic diagram of control LSI with specification.

(a)

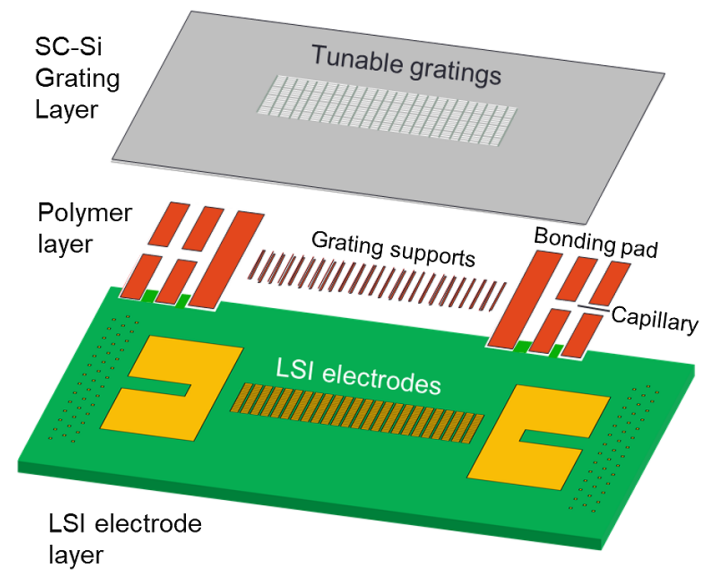

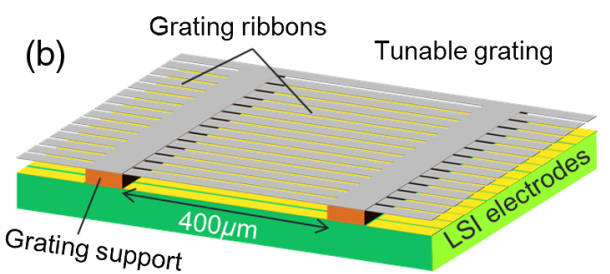

(c)

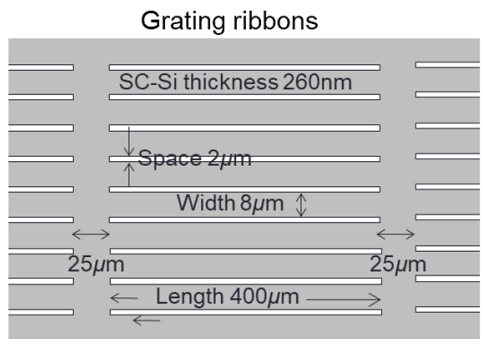

Fig. 3 (a) Schematic diagram of layered structure of decomposed tunable grating, (b) schematic diagram of grating structure, and (c) grating dimensions.

Fig. 3(a). The two bonding pads have the larger areas near the ends of LSI substrate and determine the gap between the silicon grating layer and the LSI substrate. In the polymer, a number of silica spheres with the diameter of $2.6 \mu \mathrm{m}$ are dispersed as spacers to determine the gap between them. Each bonding pad is separated into five square regions by thin spaces, which work as capillaries for filling the second polymer (epoxy polymer). Due to the dispersed silica spheres, the gap between the grating ribbons and the electrodes is reliably determined. The grating ribbons are supported in air after fabrication as shown in Fig. 3(b). The dimensions of the gratings are shown schematically in Fig. 3(c). The distance between the grating supports is $400 \mu \mathrm{m}$, which is equal to the length of the grating ribbons. The width of the grating support is about $25 \mu \mathrm{m}$. The grating ribbons are $8 \mu \mathrm{m}$ wide, $400 \mu \mathrm{m}$ long, and $260 \mathrm{~nm}$ thick. The gap between the grating ribbons is designed to be $2 \mu \mathrm{m}$ wide, and thus the period of grating is $10 \mu \mathrm{m}$ as shown in Fig. 3(c). The number of grating ribbons in each grating is 250. A 30-nm thick gold coating is assumed for reflection (not applied in this fabrication).

\section{Fabrication}

Figure 4 shows the fabrication processes using the proposed two-step polymer bonding. The two kinds of polymers (Photoneece UR3100 polymer, Toray, and Epotek 353ND polymer, Epoxy 


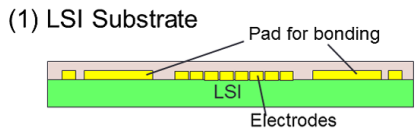

(2) passivation layer etching

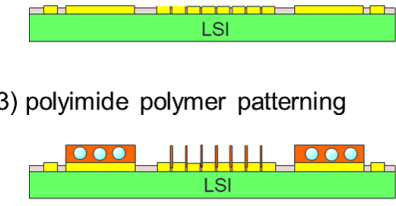

(4) SOI wafer bonding

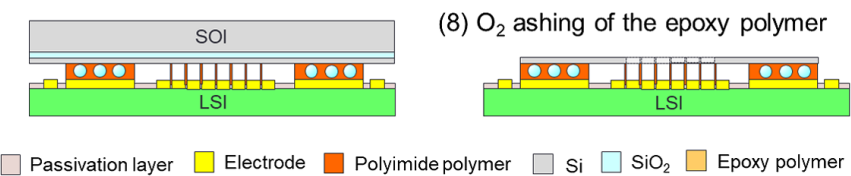

(5) Epoxy polymer filling



(6) $\mathrm{Si}$ and $\mathrm{SiO}_{2}$ etching

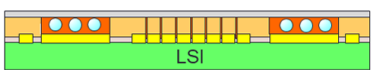

(7) Si etching to form grating

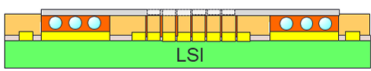

Fig. 4 Schematic diagrams of fabrication processes.

Technology) are used. The former is a negative photosensitive polyimide with a strong chemical and temperature resistance. ${ }^{21}$ The polyimide polymer is used for the formation of bonding pads and grating supports in Fig. 3. The latter epoxy polymer has a low viscosity of $500 \mathrm{cPs}$ and a low curing temperature of $90^{\circ} \mathrm{C}$. It does not have strong chemical resistance and can be easily etched by oxygen plasma. The epoxy polymer is used for filling the space between the bonded top silicon layer of SOI wafer and the electrode array of LSI substrate to avoid sticking of the top silicon layer in the subsequent processes. Finally, the epoxy polymer serves as a sacrificial layer to generate the freestanding grating ribbons. The difference in etching selectivity between these two polymers is used to leave the bonding pads and the grating supports. The selectivity of the polyimide polymer and the epoxy polymer in $\mathrm{O}_{2}$ plasma ashing process was measured to be 8 .

At process steps (1) and (2), the passivation layer $\left(\mathrm{Si}_{3} \mathrm{~N}_{4}\right)$ of electrodes of LSI substrate is etched by $\mathrm{CHF}_{3}$ (with Ar) plasma for $40 \mathrm{~min}$. The metal regions for bonding pads, grating electrodes, and grating supports are exposed. At process step (3), first, the polyimide polymer mixed with $2.6-\mu \mathrm{m}$ silica sphere spaces in ethanol is coated by spin coating on the LSI substrate. The silica spheres are used to prevent the SOI wafer from coming too close to the LSI substrate in the bonding process. The concentration of the deposited silica spheres is 800 spheres $/ \mathrm{mm}^{2}$. And then, the polymer bonding pads are formed at the bake temperature of $100^{\circ} \mathrm{C}$. The remained silica spheres are washed away in the developing process. Second, the grating supports are formed using the same polymer without silica spheres in the region of grating electrodes.

At process step (4), the SOI wafer (260-nm thick top silicon layer, $2-\mu \mathrm{m}$ thick buried oxide layer (BOX), and $200-\mu \mathrm{m}$ thick silicon substrate) is bonded on the LSI substrate at the pressure of $0.25 \mathrm{MPa}\left(50 \mathrm{~N}\right.$ piston force) and the temperature of $350^{\circ} \mathrm{C}$ for $1 \mathrm{~h}$ for the full-cure of the polymer. During the bonding, the gap between the SOI and LSI wafers is reduced by the pressure and the polymer is cured fully.

At process step (5), after the SOI wafer was bonded to the LSI substrate by the polymer, the gap between the 260-nm thick silicon layer and the electrodes on LSI substrate is filled with a low viscosity second polymer (epoxy polymer) in a vacuum chamber. Since polymer is naturally contains gas molecules, they expand and damage thin silicon layer when the sample is placed at a very low pressure of etching machine. In order to degas the polymer and fill the space using capillary force, the sample is placed in a vacuum chamber and partially connected at one side to a polymer source. ${ }^{21}$ The polymer slowly diffuses from the polymer source via a filling bridge. The filling bridge is a thin aluminum foils that works as a guide to carry the polymer from the source to the bonded wafers. The gas bubbles are restricted at the source allowing only polymer molecules to diffuse up through the narrow gap. The process takes $3 \mathrm{~h}$ to completely fill the gap of the bonded wafers from one side. This polymer filling process is crucial for supporting the grating ribbons because the thin silicon grating layer can be easily destroyed by meniscus force during wet etching process. The filled polymer is fully cured at $145^{\circ} \mathrm{C}$ for $30 \mathrm{~min}$. 
At process step (6), the silicon substrate layer of the SOI wafer is etched by $\mathrm{SF}_{6}$ plasma. Then, the BOX layer is etched by a buffered hydrofluoric acid. At process step (7), the grating array is formed. The gating layer is etched by a fast atom beam (FAB-60NL, Ebara) at process step (8), finally, the gratings are released by the sacrificial polymer etching using $\mathrm{O}_{2}$ plasma for $2 \mathrm{~h}$.

\section{Fabrication Results and Discussion}

The LSI circuits was fabricated by Toppan TDC, Japan. Figure 5 shows the optical micrograph of the fabricated LSI substrate. The electrodes for grating are magnified in the inset. The surface profile was measured with a surface profiler and is shown in Fig. 5. The height difference more than $2 \mu \mathrm{m}$ exists in the measured region and some steps are also found as shown in the measured profile. Therefore, it is important to fill the height differences with polymers for bonding the thin grating layer. An advantage of the proposed bonding method is to eliminate polishing for planarization.

The proposed bonding method was tested using an SOI wafer and a glass wafer to observe the bonded condition visibly. Figure 6(a) shows an optical micrograph of the coated polyimide polymer containing $2.6 \mu \mathrm{m}$ silica spheres. As seen in Fig. 6(a), the silica spheres are scattered randomly. The density of spheres was adjusted to be enough to keep the gap between LSI substrate and SOI wafer in the region of the bonding pads on the LSI substrate. The SOI and glass wafers were bonded at a pressure of $50 \mathrm{~N}$ and the second polymer was filled. Figure 6(b) shows an intermediate optical micrograph of filling the gap of the bonded wafers with the epoxy polymer by capillary force. The gap was gradually filled at a velocity of $120 \mu \mathrm{m} / \mathrm{min}$. Due to the capillary force and the filling bridge in vacuum, large bubbles were not trapped inside the gap, and thus it prevented from breaking the thin silicon layer during etching. The bonded interface was examined by cutting the bonded wafer. Figure 6(c) shows the cross section of the bonded wafer. The two wafers are firmly bonded with the polymers. The thickness of the polymer layer

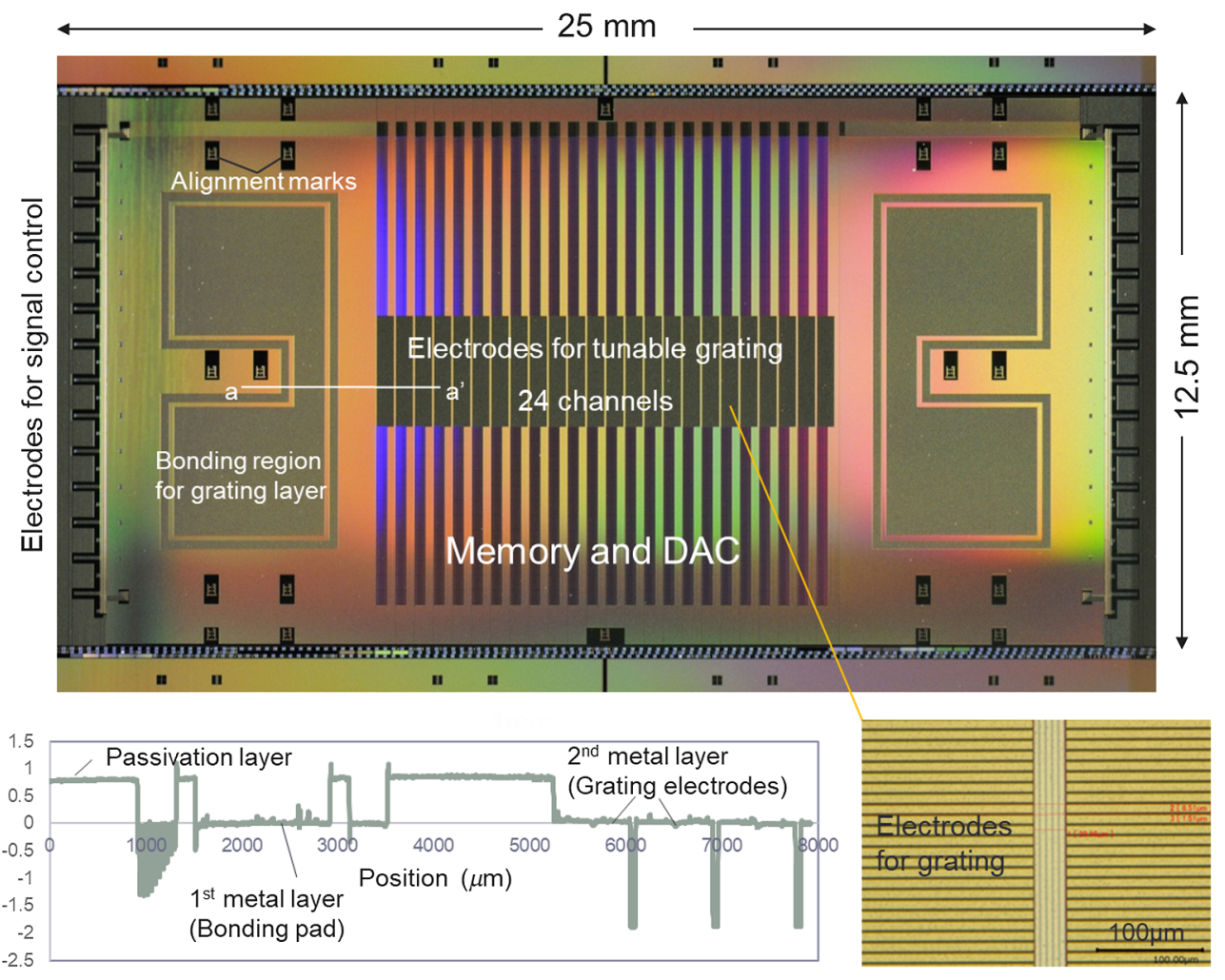

Fig. 5 Optical micrograph of the fabricated LSI substrate with the magnified image of grating electrodes and the surface profile measured along a-a' line. 


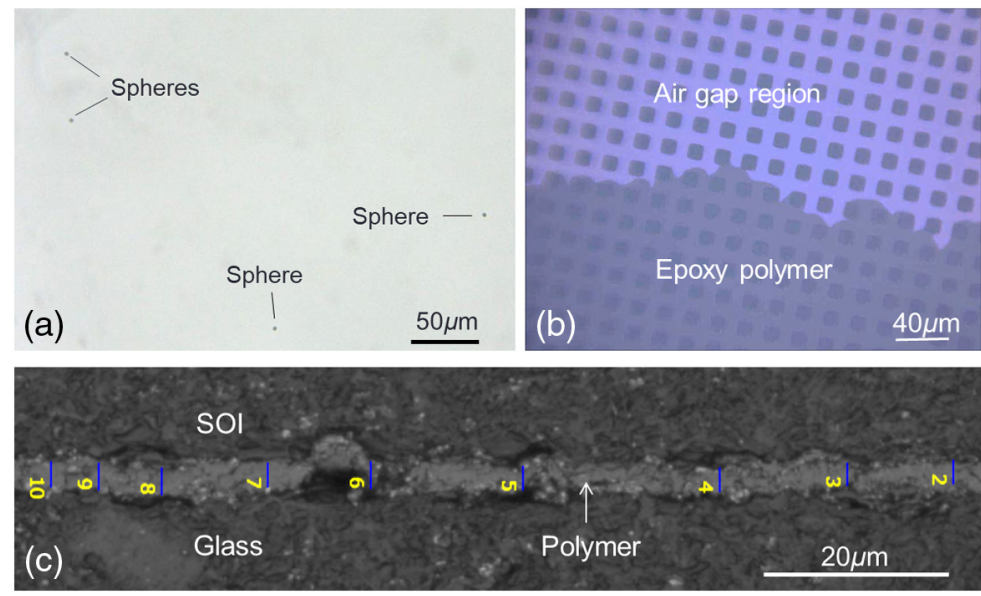

Fig. 6 (a) Optical micrograph of the coated polyimide polymer containing $2.6 \mu \mathrm{m}$ spheres, (b) optical micrograph of the SOI-glass bonded wafers, whose gap is filling with epoxy polymer, and (c) electron micrograph of the cross-section of the SOI-glass bonded wafers by the polyimide polymer. The numbers in the figure represent the positions of the gap measurement.

was measured at a number of positions. The average value of the thickness was $2.95 \mu \mathrm{m}$ with the minimum measured thickness of $2.66 \mu \mathrm{m}$. Therefore, the gap of wafers was $13.4 \%$ larger than the diameter of the dispersed spheres.

Figure 7(a) shows the whole view of the tunable gratings on the LSI substrate. The device is fabricated by the proposed bonding method. The magnified images of the grating ribbons are shown in the insets of Fig. 7(a). The spaces between the grating ribbons are somewhat widened in this sample, which are caused by an excess etching and the precision limit of $1 \mu \mathrm{m}$ in our photolithography system. We fabricated devices one by one. The yield of grating fabrication was roughly the half of the fabrications under our laboratory condition with homemade process machines, which will be much improved by optimizing process conditions and device handling.

Figure 7(b) shows the surface profile of a grating area measured by an optical interferometer (MSA-500, Polytec). The height difference between red (high) and blue (low) regions is about $2.5 \mu \mathrm{m}$. Although there are some defects caused in our home-made process setup, the height fluctuation is approximately $\pm 15 \mathrm{~nm}$ in the area without defects. In the whole grating area, it was difficult to fabricate all the gratings without defects in our laboratory. The defects may be caused by the surface contamination in the home-made setup and the sticking of ribbons to substrate by the charge-up in the final ashing process.
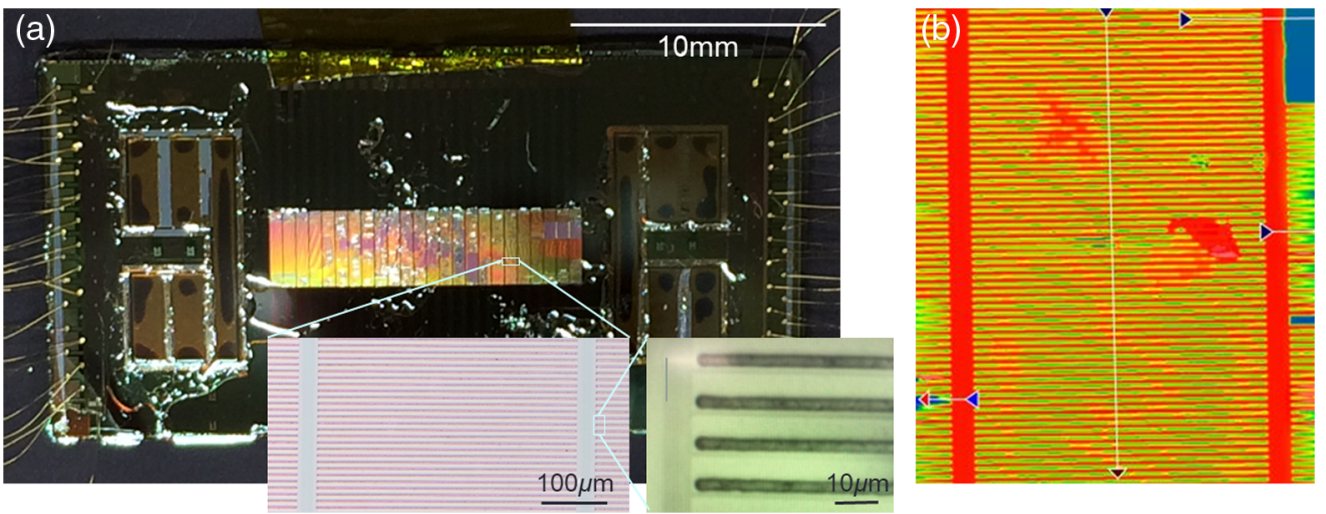

Fig. 7 (a) Optical micrograph of fabricated tunable gratings on LSI substrate with magnified images of gratings and (b) surface profile of a grating area measured by optical interferometer. 


\section{Operation Results and Discussion}

The motion characteristics of the fabricated grating ribbons were examined experimentally. The static displacement and the deflection of grating ribbons were measured under the microscopic objective of the interferometer (MSA-500, Polytec) by increasing the voltage applied to a grating ribbon. The dynamic motion of a grating ribbon, which corresponded to the velocity, was measured by a laser Doppler vibrometer (OFV-5000, Polytec) as a function of time by applying a step voltage. First, the static displacements of the grating ribbons were measured. Figure 8(a) shows the displacement at the center of a ribbon measured as a function of the voltage generated by the LSI circuit. The displacement increases nearly quadratically as a function of the voltage from 0 to $5 \mathrm{~V}$. The maximum displacement is $850 \mathrm{~nm}$ at $5 \mathrm{~V}$, which is larger than a half of a typical communication wavelength of $1550 \mathrm{~nm}$ and thus enough to generate $2 \pi$ phase delay in reflection.

The grating ribbons generally deflected slightly downward under the initial condition. The downward defection was considered to be generated by the rotation moments at the fixed ends of grating ribbons caused by the polymer, where the grating ribbons suffered roughly $3 \mathrm{MPa}$ tensile stress.

Figure 8(b) shows the distribution of the displacement of the grating ribbon along the length of the ribbon. The grating ribbon deflects downward due to the attractive electrostatic force. The displacement distribution is approximately quadratic, which is explained by the deformation of a both-end fixed beam. The displacements of grating ribbons were somewhat dependent on the fabricated samples. The quadratic deflection after applying voltage yields different grating depths along the length of grating ribbon. Therefore, to reflect incident light beam at a welldefined reflection phase, it is preferable to focus the light beam on the center parts of grating ribbons. It is noted that the height of grating ribbon was continuously varied with keeping the flat ribbon shape in the case of the leverage bending mechanism of polychromator. ${ }^{11}$

The influence of grating ribbon deflection by electrostatic force on the reflected light intensity was investigated numerically. Under the maximum deflection condition of grating ribbon corresponding to the displacement of a half of the wavelength, the reflected light intensity was decreased to about $30 \%$ of the incident intensity when the grating ribbon was illuminated uniformly. This was caused by the interference of the light reflected by the deflected ribbon. In order to prevent from decreasing the reflected light intensity to $<80 \%$, the illumination area of grating

(a)

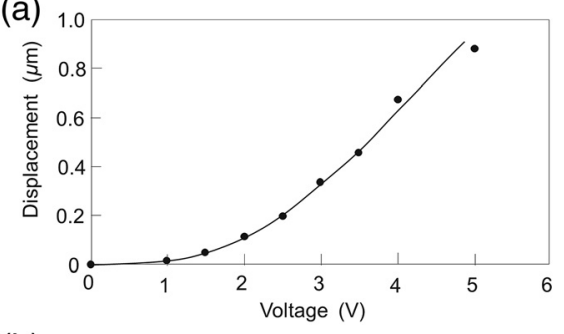

(c)

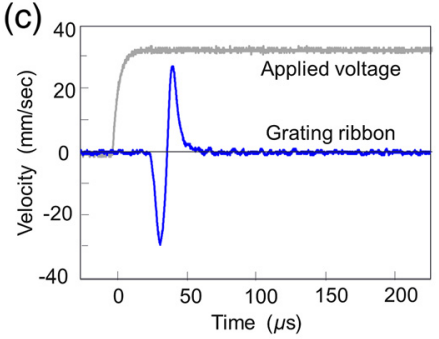

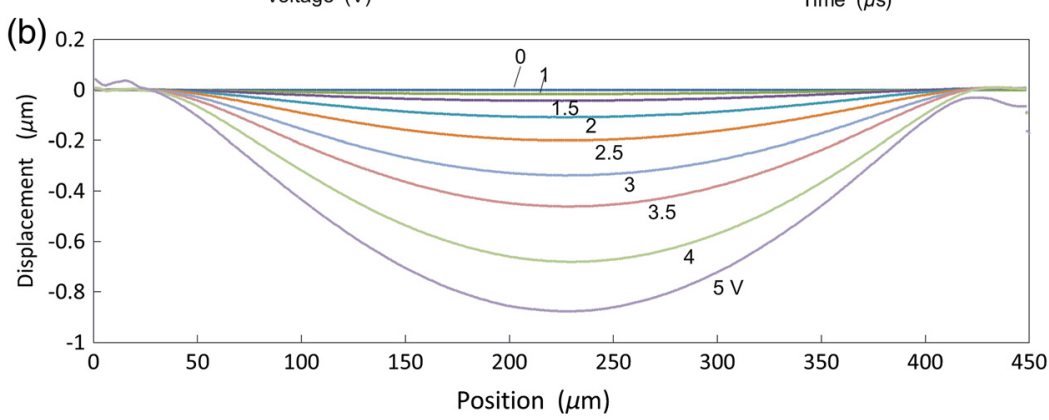

Fig. 8 (a) Displacement of a grating ribbon at the center measured as a function of voltage, (b) deflection of the grating ribbon measured as a function of position along the length of ribbon with applied voltage as parameter, and (c) velocity of the grating ribbon at the center measured as a function of time. 
ribbon should be limited to the central region of ribbon smaller than $45 \%$ of the ribbon length by focusing the incident light with lens.

Figure 8(c) shows the velocity of a grating ribbon at the center as a function of time after applying a step voltage of $5 \mathrm{~V}$ through the LSI circuit. In the Doppler vibrometer measurement, it was usually difficult to detect low frequency components of velocity such as de component. Therefore, the measured velocity did not include the low frequency components and the grating ribbon displacement including dc displacement was not obtained by integrating the measured velocity. The response time to the applied step voltage was roughly estimated from the duration of the velocity signal. The response time of the grating ribbon for reaching a stable position is $\sim 70 \mu \mathrm{s}$, which is larger than the LSI signal processing time of about $10 \mu \mathrm{s}$. Therefore, the setting time of grating ribbon is mainly determined by the response time of the mechanical motion of grating ribbon.

The controllability of the fabricated grating by the LSI circuit was examined experimentally using several programs. The programs are made in a personal computer and send to the fabricated grating through SPI communication to apply a set of voltages to respective grating ribbons. Figure 9 shows the examples of the tested results. Figures 9(a) and 9(b) show the interferometric fringe images of the grating surface at the initial condition without voltage (one-level) and the grating surface where the voltages $<5 \mathrm{~V}$ are applied alternately to the grating ribbons (two-level), respectively. In Fig. 9(a), the fringe is continuously observed so that the grating surface is planar. In Fig. 9(b), the interferometric fringe is discontinuous and the image period is twice larger than the grating period. In the interferometric images, the object is imaged with the interference light intensity at each position of the object. A small tilt of flat object generates a fringe image composed of dark and bright lines with the period corresponding to the tilt as shown in Fig. 9(a). If there is a height distribution in the object, the positions having the same height along fringe appear at the same fringe intensity. Therefore, when the height of gating ribbon decreases, the intensity of fringe changes to the intensity corresponding to the height, which introduces a fringe shift at the lowered positions. And thus, the fringe becomes discontinuous along the fringe as shown in Fig. 9(b). Therefore, it is confirmed that the grating ribbons are addressed by the LSI circuit, and the respective heights of ribbons are modulated alternately.
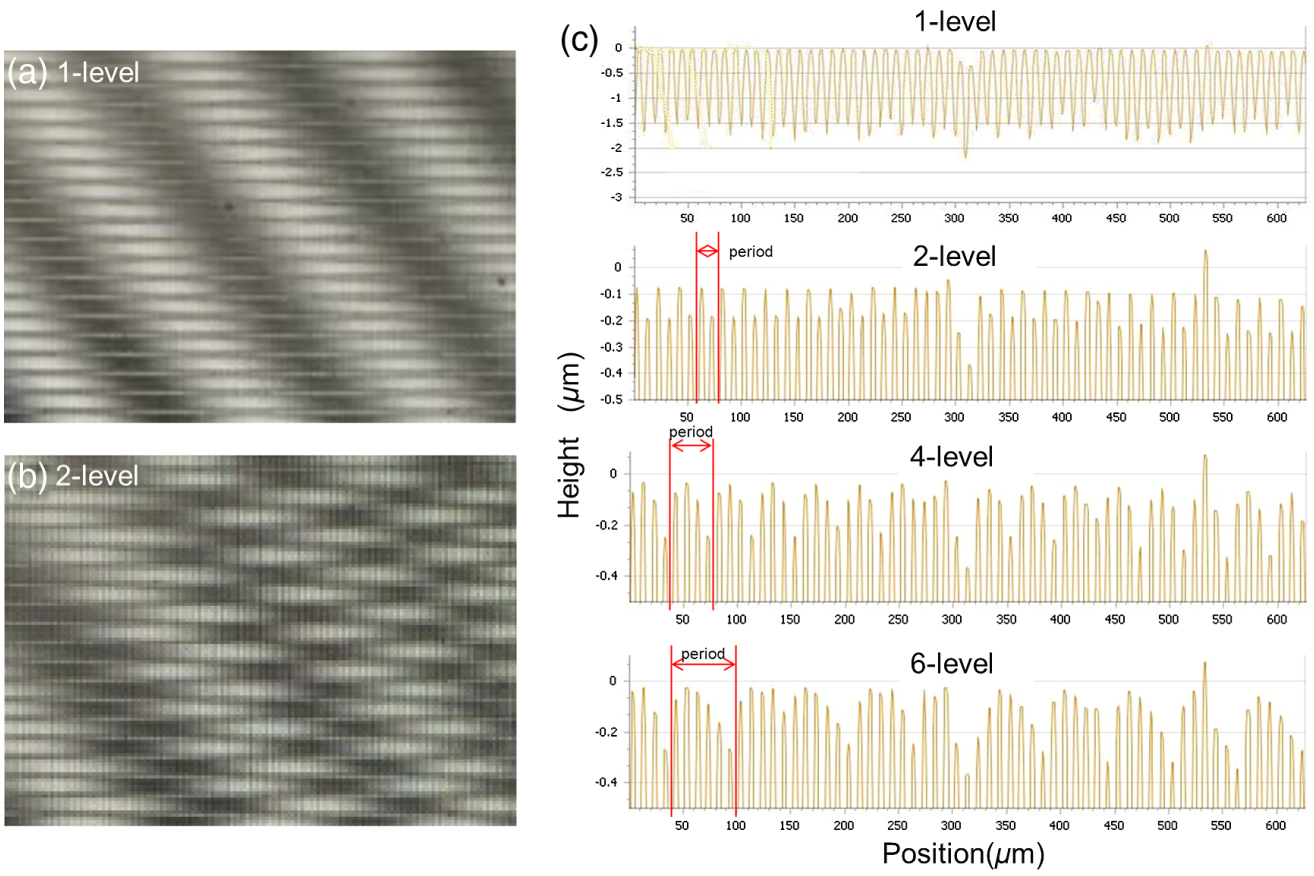

Fig. 9 Interferometric fringe images observed in (a) one-level and (b) two-level grating arrangements, respectively. (c) The heights of grating ribbons measured as a function of position in one-, two-, four-, and six-level arrangements. 
A set of saw-toothed voltages with different levels were generated in the LSI circuit and were applied to the respective grating ribbons. The heights of the grating ribbons were measured with the interferometer (MSA-500, Polytec) at the centers of the ribbons in the direction vertical to the grating ribbons. The measured results are shown in Fig. 9(c) in the respective level arrangements. In the one-level arrangement of grating ribbons, the ribbons are all at the initial positions with applying zero voltages to the ribbons. All the grating ribbons are located at the highest positions with a few defects of ribbons. In the two-level arrangement, a set of alternating voltages are applied to the grating ribbons, and the height distribution is measured. As shown in Fig. 9(c), the heights of ribbons change up and down alternately with varying position. The height difference between the two levels is $\sim 120 \mathrm{~nm}$ in this experiment. In the four-level arrangement, four grating ribbons are included in a period of grating. Although the applied voltages to the grating ribbons decrease similarly to the voltage dependence of the displacement shown in Fig. 8(a), the heights of the grating ribbons are not decreased corresponding to the voltages as shown in Fig. 9(c). This can be explained by the stray electrostatic fields from the neighboring electrodes. The left-most ribbon with $0 \mathrm{~V}$ in a period of four-level grating is attracted by the highest voltage acting on the neighboring electrode, i.e., the right-most electrode in the right-side period as seen in the four-level result of Fig. 9(c). In the six-level arrangement, the measured height distribution is shown in Fig. 9(c). The six different height levels are generated in a period of the saw-toothed waveform. The heights of grating ribbons are, however, influenced from the neighboring electrodes similarly to the four-level arrangement. From those measurements, it is found that the heights of the grating ribbons can be controlled by the applied voltages generated by the LSI circuit. However, it is necessary to adjust the voltages applied to the electrodes considering the influences from neighboring electrodes, which should be determined in advance.

To test the beam steering by diffraction, the diffraction patterns of the tunable grating were observed at a distance of about $30 \mathrm{~cm}$ from the grating using a Fourier transformation lens. The laser light at the wavelength of $406 \mathrm{~nm}$ was used for the test of gratings. Since the grating ribbon was not coated with a gold reflection film, the grating ribbon was transparent at $1550 \mathrm{~nm}$. The transmitted light often generates noise by multiple reflection. At $406 \mathrm{~nm}$, the reflectivity is about $50 \%$ and the transmitted light is not generated because silicon is absorptive at this wavelength. The light at $406 \mathrm{~nm}$ was also useful for observation by the eyes. Figure 10(a) shows the diffraction patterns in the grating arrangements of one-level, two-level, and three-level, respectively. In the one-level arrangement of grating ribbons, the two diffraction spots are observed with the zeroth-order spot as shown in Fig. 10 (a). The two-diffraction spot with the nearly equal intensities is caused by the grating ribbons located in the same height with the gap between grating ribbons. The angles of the diffraction beams are calculated to be about $2.3 \mathrm{deg}$, which is corresponding to the period of $10 \mu \mathrm{m}$ at the wavelength. The large intensity in the zeroth-order is caused mainly by the fact that the diameter $(\sim 2 \mathrm{~mm})$ of the incident laser beam is larger than the grating area, and partially reflected from the region outside the controlled grating.

(a)

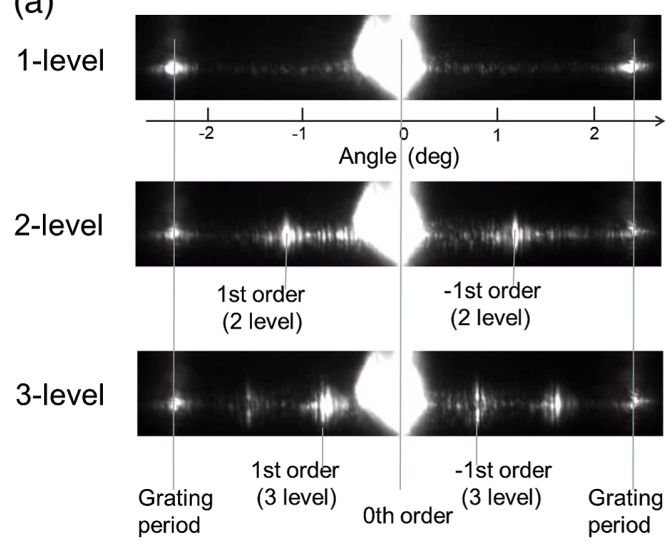

(b)

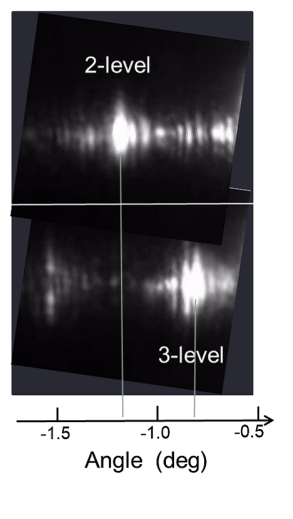

Fig. 10 (a) Diffraction patterns in different-level arrangements of grating ribbons and (b) position switching of one-order diffraction beam by program. 
In addition, the diffraction efficiency of the fabricated grating is low ( $~ 50 \%)$ due to the uncoating of gold film.

In the two-level arrangement of grating, as seen in Fig. 10(a), the four diffraction spots are observed except for the zeroth-order spot. The inner two diffraction spots are generated by the first-order diffraction in the two-level arrangement of grating ribbons. Those diffraction spots appear at the center positions between the zeroth-order spot and the first-order spots in the onelevel arrangement. The diffraction angle is $1.2 \mathrm{deg}$, which is a half of the value in the one-level arrangement.

In the three-level arrangement of grating ribbons, the diffraction spots appear as shown in Fig. 10(a). The spots beside the zeroth-order spot are the first-order diffraction spots in the threelevel arrangement. Those spots are located at the distance of one-third of the distance between the spot of the grating period and the zeroth spot because three ribbons compose one period of the grating profile in the three-level arrangement. The intensity of the +1 -order beam in the three-level arrangement is larger by a factor of 4 than that in the -1-order. Although the intensity in the -1-order diffraction is not zero, the larger intensity of the blazed grating appears as predicted theoretically.

The switching of the position of the +1 -order diffraction beam is tested using a program for LSI circuit. As shown in Fig. 10(b), the position of the +1 -order diffraction beam in the twolevel arrangement is shifted to that in the three-level arrangement by the angle shift of $\sim 0.4 \mathrm{deg}$. Although the diffraction property was not ideal, the proposed tunable grating bonded on LSI circuit operated.

\section{Conclusions}

The multileveled tunable silicon grating on the LSI substrate was designed and fabricated by the two-step polymer bonding method at the temperature of $350^{\circ} \mathrm{C}$. The LSI substrate generated the respective voltages from 0 to $5 \mathrm{~V}$ to vary the heights of the grating ribbons addressed by 6-bit-digital signals. In the fabrication, the photosensitive polyimide polymer, in which $2.6-\mu \mathrm{m}$ diameter silica spheres were dispersed, was used for patterning the bonding pads of gratings. The gap between the silicon layer for gratings and the LSI substrate was determined by the silica spheres under the applied pressure of $0.25 \mathrm{MPa}$. The measured value of the gap was $2.95 \mu \mathrm{m}$ in average. Another epoxy polymer filled the space underneath the grating ribbons. The epoxy polymer was finally etched as sacrificial layer to make the bonded silicon layer freestanding as grating ribbons. The tunability of the grating was tested experimentally using the program of SPI communication. The grating ribbons were attracted to the electrodes of LSI substrate for generating the multilevel arrangements of grating ribbons. The generation of the height distribution of grating ribbons was confirmed. However, the heights of grating ribbons were influenced by the neighboring electrodes. It is necessary to compensate the control values in the program. In the preliminary experiment, the position of the first-order diffraction beam was switched by the program, which will be useful for developing a WSS in future.

\section{Acknowledgments}

Authors thank J. Oguri and N. Kagi for their useful advice, and also thank A. Endo, M. Sakaki, and T. Sekiya for their experimental works. This study was supported by Special Coordination Funds for Promoting Science and Technology (JST: Japan Science and Technology Agency, Japan), Creation of Innovation Center for Fusion of Advanced Technology (MEXT: Ministry of Education, Culture, Sports, Science and Technology, Japan), and KAKENHI (JSPS: Japan Society for the Promotion of Science, Japan). The fabrications were carried out at the Micro/ Nano-Machining Research and Education Center Tohoku University.

\section{References}

1. X. Li et al., “Tunable blazed gratings," J. Microelectromech. Syst. 15(3), 597-604 (2006).

2. C. W. Wong et al., "Analog tunable gratings driven by thin-film piezoelectric microelectromechanical actuators," Appl. Opt. 42(4), 621-626 (2003). 
3. Y.-C. Yung and K. Kuribayashi, "Nanoimprint strain-controlled elastomeric gratings for optical wavelength tuning," Appl. Phys. Lett. 86(16), 161113 (2005).

4. Y. Wang, Y. Kanamori, and K. Hane, "Pitch-variable blazed grating consisting of freestanding silicon beams," Opt. Express 17(6), 4419-4426 (2009).

5. D. M. Burns and V. M. Bright, "Development of microelectromechanical variable blaze gratings," Sens. Actuators A 64, 7-15 (1998).

6. H. Sagberg et al., "Micromechanical gratings for visible and near-infrared spectroscopy," IEEE J. Sel. Top Quantum Electron. 10(3), 604-613 (2004).

7. Y. Hongbin et al., "An electromagnetically driven lamellar grating based on Fourier transform microspectrometer," J. Micromech. Microeng. 18, 055016 (2008).

8. O. Solgaard, F. S. A. Sandejas, and D. M. Bloom, "Deformable grating optical modulator," Opt. Lett. 17(9), 688-690 (1992).

9. K. Ishikawa et al., "Sub-nm order fluctuation control of narrowed pitch $2 \mathrm{~K}-\mathrm{GxL}$ device for high contrast," in Proc. Opt. MEMS \& Nanophotonics, pp. 45-46 (2009).

10. B.-W. Yoo et al., "A $32 \times 32$ optical phased array using polysilicon sub-wavelength highcontrast-grating mirrors," Opt. Express 22(16), 19029-19039 (2014).

11. G. B. Hocker et al., "The polychromator: a programmable MEMS diffraction grating for synthetic spectra," in Solid-State Sens. and Actuator Workshop, Hilton Head Island, pp. 89-91 (2000).

12. D. M. Marom et al., "Wavelength-selective $1 \times \mathrm{K}$ switches using free-space optics and MEMS micromirrors: theory, design, and implementation," J. Ligthwave Technol. 23(4), 1620-1630 (2005).

13. S. Serati and J. Stockley, "Advanced liquid crystal on silicon optical phased arrays," Aerosp. Conf. Proc. 3, 1395-1402 (2002).

14. S. Qin et al., "Liquid crystal-optical phased arrays (LC-OPA)-based optical beam steering with microradian resolution enabled by double gratings," Appl. Opt. 58(15), 4091-4098 (2019).

15. W. Jung, U. Krishnamoorthy, and O. Solgaard, "High fill-factor two-axis gimbaled tip-tiltpiston micromirror array actuated by self-aligned vertical electrostatic combdrives," J. Microelectromech. Syst. 15(3), 563-571 (2006).

16. M. Lapisa, G. Stemme, and F. Niklaus, "Wafer-level heterogeneous integration for MOEMS, MEMS, and NEMS," IEEE J. Sel. Top. Quantum Electron. 17(3), 629-644 (2011).

17. A. Witvrouw et al., "11-megapixel CMOS-integrated SiGe micromirror arrays for high-end applications," J. Microelectromech. Syst. 19(1), 202-214 (2010).

18. F. Niklausa et al., "Adhesive wafer bonding," J. Appl. Phys. 99(3), 031101 (2006).

19. F. Zimmer et al., "One-megapixel monocrystalline-silicon micromirror array on CMOS driving electronics manufactured with very large-scale heterogeneous integration," J. Microelectromech. Syst. 20(3), 564-572 (2011).

20. T. Matsumura et al., "Multi-band radio-frequency filters fabricated using polyimide-based membrane transfer bonding technology," J. Micromech. Microeng. 20, 095027 (2010).

21. S. Chernroj et al., "Fabrication and evaluation of single-crystal-silicon tunable grating using polymer-based membrane transfer bonding process," IEEJ Trans. Sens. Micromach. 135(9), 361-366 (2015).

22. J. W. Goodman, Introduction to Fourier Optics, McGraw-Hill, San Francisco (1968).

Tomohiro Suzuki received his MS degree in mechanical engineering at Tohoku University, Japan, in 2016. He joined Canon Inc.

Sawasdivorn Chernroj received his BS degree in electronics and communication engineering at Sirindhorn International Institute of Technology, Thammasat University, Thailand. From 2012 to 2015 he received support from a Panasonic Scholarship. He received his MS degree in mechanical engineering at Tohoku University, Japan, in 2015.

Takashi Sasaki received his $\mathrm{PhD}$ in mechanical engineering from Tohoku University, Japan, in 2012. Since 2012, he has been an assistant professor with the Graduate School of Mechanical Engineering, Tohoku University, where he is currently engaged in research and development of microelectromechanical systems. 
Hiroshi Matsuura received his $\mathrm{PhD}$ in mechanical engineering from Tohoku University, Japan in 2007. He has been a professor in the Department of Mechanical Engineering, Tohoku Gakuin University, Japan.

Kazuhiro Hane received his $\mathrm{PhD}$ in electronics from Nagoya University, Japan, in 1983. From 1985 to 1986, he was a visiting researcher with the National Research Council of Canada. Since 1994, he has been a professor with the Gradual School of Mechanical Engineering, Tohoku University, Japan, where he is currently engaged in research and development of optical microsensors and optical microelectromechanical systems. 